\title{
Obtención de $\kappa$-Carragenano y $\lambda$-Carragenano a partir de macroalga Chondracanthus chamissoi y su aplicación en la industria alimentaria
}

(1) Norma Salas De La T.
${ }^{(2)}$ César Córdova C.
${ }^{(3)}$ Edmundo Estrada A.

Recepción: Mayo de 2008 / Aceptación: Setiembre de 2008

\begin{abstract}
RESUMEN
La explotación de algas productoras de carragenanos se intensifican a partir de los 80 .

La obtención de $\mathrm{K}$-carragenano y $\lambda$-carragenano está en función de la selección adecuada de algas, identificación correcta de las fases de vida, contro de parámetros como temperatura, $\mathrm{pH}$, tiempo y concentración de soluciones.

El proceso de extracción se basa en: solubilidad en agua caliente e insolubilidad en solventes orgánicos polares.

La fase gametofita es punto de partida para la producción de k-carragenano y la fase esporofita se orienta a la producción de $\lambda$-carragenano.

La capacidad gelificante ( $k$-carragenano) y el comportamiento viscosante ( $\lambda$-carragenano) de los carragenanos son características que se aplican para gelificar o espesar sistemas acuosos.
\end{abstract}

Palabras clave: Carragenanos, Chondracanthus, Polisacáridos, Fracción gelificante.

ACQUisition of K- AND $\lambda$ - CARRAGEENAN FROM MACROALGAE CHONDRACANTHUS CHAMISSOI AND ITS APLICATION IN THE FOOD INDUSTRY

\section{ABSTRACT}

The exploitation of algae producing carrageenan are intensified since the $80^{\prime}$.

Obtaining $\mathrm{k}$-carrageenan and $\lambda$-carrageenan is a function of the proper selection of algae, correct identification of the stages of life, control of parameters such as temperature, $\mathrm{pH}$, time, concentration of solutions.

The extraction process is based on: soluble in hot water and insolubility in polar organic solvents.

Gametophyte phase is a starting point for the production of $\mathrm{k}$-carrageenan and phase sporophitic is geared to the production of $\lambda$ - carrageenan.

The ability gelling ( $\mathrm{k}$-carrageenan) and performance viscosante ( $\lambda$-carrageenan) of carrageenan are characteristics that apply to gelificar or thicken aqueous systems.

Key words: Carrageenan, Chondracanthus, Polysaccharides, Fraction gelling.

\section{INTRODUCCIÓN}

Las algas marinas se caracterizan por su riqueza en oligoelementos, proteínas, vitaminas, iodo, fósforo, potasio, que no son frecuentes en otros alimentos; pero su gran riqueza está en los hidrocoloides que posee, considerados nutracéuticos.

Las algas rojas contienen polisacáridos complejos denominados ficocoloides (carragenanos) cuyas propiedades dependen en gran medida de los cationes a los que se asocian, así pueden formar geles firmes en presencia del catión potasio ( $\mathrm{k}$-carragenano) o fracciones no gelificantes ( $\lambda$-carragenano) debido a su alto grado de sulfatación.

Chondracanthus chamissoi, alga rodophyta que abunda en la Costa Sur del Pacífico de aguas templadas (Bahía de San Nicolás, Laguna Grande, Playa Mendieta, Pisco), de la que se extrae K-carragenano, $\lambda$ carragenano, I-carragenano y $\mu$-carragenano, poseen propiedades antivirales, antilipogénicas e hipolipemiantes, según diversos autores; así Baba y Colaboradores (3) lograron inhibir la replicación de algunos virus en dextran sulfato, posteriormente Fuertes $C$. (7) propone que todo polisacárido sulfatado tiene efectos similares o superiores frente a diversos virus con una ventaja manifiesta para los polisacáridos naturales. Posteriormente se ensaya con la fracción soluble $\lambda$-carragenano extraída de Chondracanthus chamissoi en su fase femenina y tetraspórica, y se observa que posee gran actividad inhibitoria de la replicación viral del virus de inmunodeficiencia adquirida, VIH.

La fracción insoluble gametofita k-carragenano, muestra menos actividad inhibitoria viral.

En el Perú no existe industria procesadora de carragenanos siendo importada de Estados Unidos de Norteamérica, Japón, Francia, Canadá, Dinamarca y Chile.

En el Perú existen tres empresas que se dedican a la extracción y comercialización del alga fresca, refrigerada, congelada y seca; éstas son: Crosland Técnica S.A., Peruvian Seaweeds S.R.L. y Vidal Vidal Elio.

Chondracanthus chamissoi es extraída del Submareal y exportada principalmente a países asiáticos (Japón) para su transformación y consumo directo (Gil-Kodaka, 2002).

\footnotetext{
Directora de la E.A.P. de Ingeniería Agroindustrial- UNMSM. nsalasd@hotmail.com

Responsable del Lab. de Ficología - Fac. C. Biológicas. ccordovac@unmsm.edu.pe

Docente Asociado del D.A.Química Inorgánica edmundoestrada2000@yahoo.com

Facultad de Química, Ingeniería Química e Ingeniería Agroindustrial-Facultad de Ciencias Biológicas de la Universidad Nacional Mayor de San Marcos, Lima-Perú.
} 


\section{PARTE EXPERIMENTAL}

El proceso de extracción de carragenanos se basa en dos propiedades fundamentales de los carragenanos:

- Su solubilidad en agua caliente.

- Su insolubilidad en solventes orgánicos polares.

Las algas Chondracanthus chamissoi con estructuras reproductivas maduras se recolectaron del Submareal en la Bahía de Paracas por los miembros del grupo de investigación que encabeza el profesor de la UNMSM Córdova Castañeda, y trasladadas a Planta Piloto de la Facultad de Química e Ingeniería Química para ser separadas en sus fases de vida:

- Fase gametofito femenino

- Fase gametofito masculino

- Fase tetraspórica

- Cuarto grupo (mezcla de las fases), 4to G

\section{Etapa de preparación de las algas}

Las algas clasificadas en sus fases de vida son lavadas para remover materia extraña, y enjuagadas repetidamente con agua destilada. Posteriormente, se somete a secado hasta peso constante y por fases. Se procedió luego a la molienda grosera.

Las algas molidas son sometidas a hidratación con agua destilada y agitación constante. Se enjuagó repetidamente para extraer pigmentos y sales, así tenemos las preparadas para la extracción.

Figura N.1: Preparación de algas

\begin{tabular}{|c|} 
Algas rojas (Chondracanthus chamissoi) \\
\hline $\begin{array}{c}\text { Separación de las algas de fase de vida } \\
\text { gametofítica de las esporofitas }\end{array}$ \\
\hline Prueba con resorcinol-acetal \\
\hline Secado del alga (5 g aprox.) \\
\hline T: $60^{\circ} \mathrm{C}$ \\
\hline \\
\hline Lavar las algas repetidamente \\
\hline
\end{tabular}

FUENTE: Elaboración propia.

\section{Etapa de Extracción de $\mathrm{k}$-carragenano (Tratamiento Alcalino en Frío)}

Para el proceso de extracción se ha tomado el método modificado de Craige y Leigh (1978), siendo adaptado con algunas variantes por los miembros del proyecto.

Las algas preparadas son tratadas con hidróxido de potasio al 6\% P/P con agitación vigorosa para promover la extracción total de polisacárido. Deberán permanecer en la solución alcalina durante 24 horas. Evacuar parte de la solución alcalina dejando un remanente que cubra solamente las algas por otro tiempo igual.

Se retiran las algas de la solución alcalina y se observa el agua remanente, presenta un matiz amarillo intenso.

Seguidamente, se colocan las algas en sacos de muselina o nylon de doble tramo y se sumergen en agua destilada repetidamente y se deja por 12 horas.

Estirar las algas en forma uniforme en bandejas de acero inoxidable y llevar a congelación por 12 horas y descongelarlas. Repetir este paso dos veces.

Llevar a estufa a $42-45^{\circ} \mathrm{C}$ hasta peso constante. Pesar el carragenano semi refinado.

\section{Figura N. 2: Tratamiento alcalino}

Colocar muestras de algas preparadas en $400 \mathrm{ml}$ de $\mathrm{KOH}$ al $6 \% \mathrm{P} / \mathrm{P}(24 \mathrm{~h})$

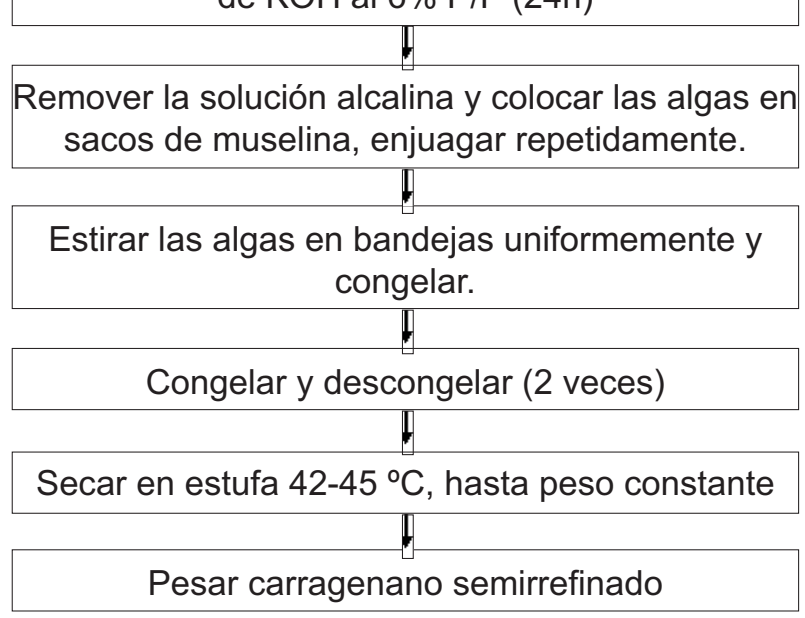

FUENTE: Adaptado de Cristian Bulboa Contador - Universidad Católica del Norte, Coquimbo - Chile. 
Figura N.․ 3: Fraccionamiento

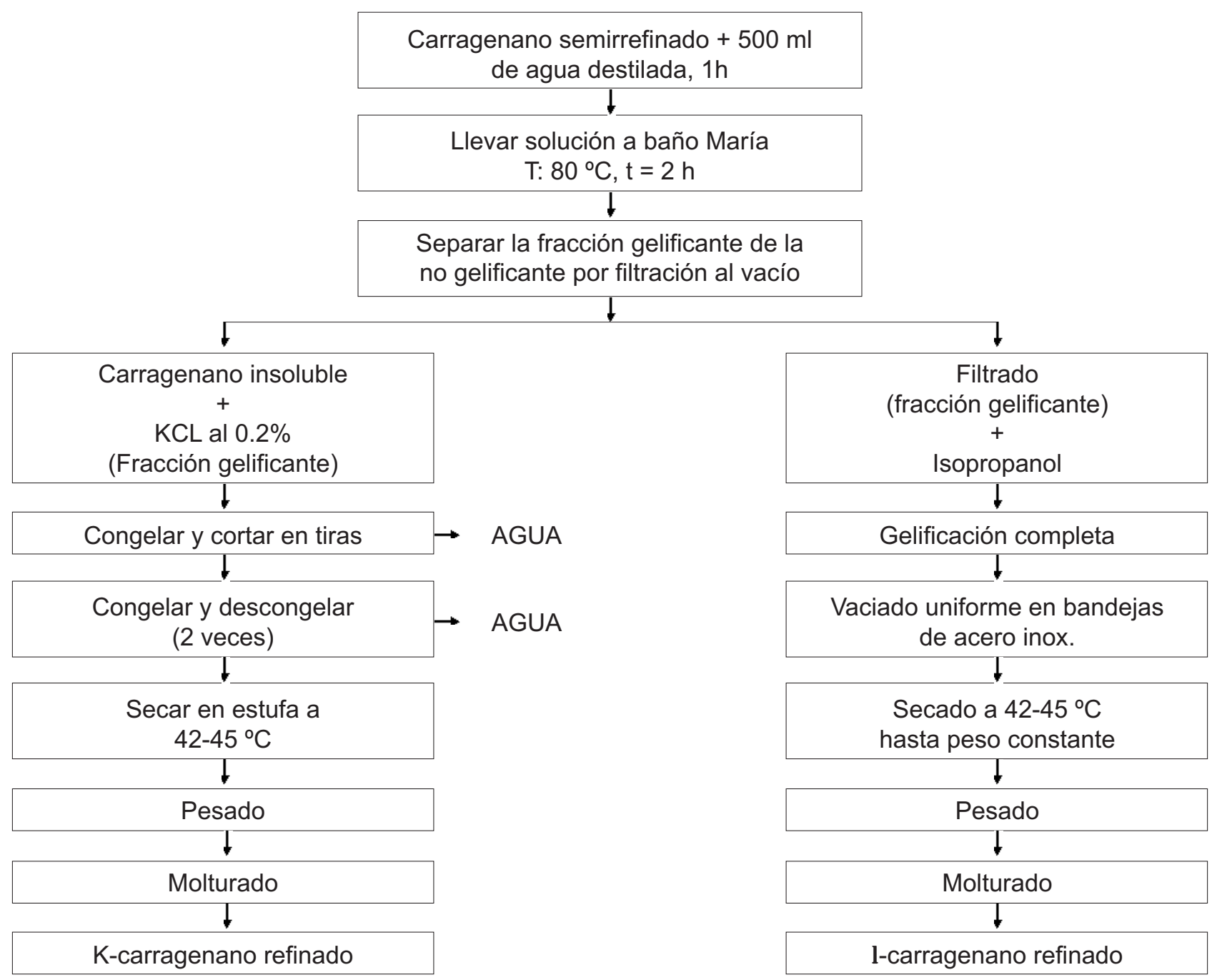

FUENTE: Adaptado de Cristian Bulboa Contador. Universidad Católica del Norte, Coquimbo, Chile.

\section{Etapa de Fraccionamiento}

El carragenano semi refinado es hidratado con agua destilada y dejar en reposo. Luego, se lleva a baño maría a $80^{\circ} \mathrm{C}$ durante dos horas. Separar las fases por filtración al vacío. La fracción gelificante es insoluble y es retenido en el filtro, esta fracción es tratada con cloruro de potasio al $0,20 \% \mathrm{P} / \mathrm{P}$ y posteriormente se extiende en bandejas de acero inoxidable para llevarla a congelación. Congelar y descongelar (2 veces). Secar en estufa a $42-45^{\circ} \mathrm{C}$ por 24 horas aproximadamente. El polvo obtenido de color blanco debe pesarse para calcular el rendimiento de K-carragenano.

Por otro lado, el filtrado (fracción no gelificante), es tratada con isopropanol coagulando completamente. Esta fracción es extendida en bandejas de acero inoxidable y llevadas a secado a $45^{\circ} \mathrm{C}$ hasta peso constante. Pesar el $\lambda$-carragenano para efectos de hallar el rendimiento. 


\section{RESULTADOS}

Tabla $\mathrm{N}^{\circ}$. 1: Rendimiento de carragenano semi refinado

\begin{tabular}{|c|c|c|c|}
\hline Muestra & Peso de Muestra & Peso Carragenano semirrefinado & Rendimiento \\
\hline$(\%)$ & & & \\
\hline $\mathrm{N} .{ }^{\circ} 1(+)$ & $5,000 \mathrm{~g}$. & 2,6213 & 52,4 \\
\hline $\mathrm{N} .{ }^{\circ} 2\left({ }^{\curvearrowright}\right)$ & 5,000 & 2,2923 & 45,8 \\
\hline $\mathrm{N} .{ }^{\circ} 3(\otimes)$ & & & \\
\hline 4,6240 & 3,0383 & 65,7 & 38,2 \\
\hline $\mathrm{N} .{ }^{\circ} 4(4$ to G) & 5,000 & 1,9109 & \\
\hline
\end{tabular}

Tabla N. 2: Peso de K-carragenano y $\lambda$-carragenano

\begin{tabular}{|c|c|c|c|c|}
\hline MUESTRA & PESO DE MUESTRA & $\begin{array}{c}\text { PESO carragenano } \\
\text { semirrefinado }\end{array}$ & $\begin{array}{c}\text { PESO } \\
\text { K-carragenano }\end{array}$ & $\begin{array}{c}\text { PESO } \\
\text {-carragenano }\end{array}$ \\
\hline $\mathrm{N} .{ }^{\circ} 1(+$ - $)$ & $5,000 \mathrm{~g}$. & 2,6213 & 1,8138 & 0,8075 \\
\hline $\mathrm{N} .{ }^{\circ} 2\left(\jmath^{\lambda}\right)$ & $5,000 \mathrm{~g}$. & 2,2923 & 1,7690 & 0,5233 \\
\hline $\mathrm{N} .{ }^{\circ} 3(\otimes)$ & & & & \\
\hline 4,6240 & 3,0383 & 0,9445 & 2,0938 & \\
\hline $\mathrm{N} .{ }^{\circ} 4(4$ to G) & 5,000 & 1,9109 & 1,1475 & 0,7633 \\
\hline
\end{tabular}

Tabla N. ${ }^{\circ}$ 3: Rendimiento de K-carragenano

\begin{tabular}{|c|c|c|c|}
\hline MUESTRA & PESO MUESTRA & $\begin{array}{c}\text { PESO } \\
\text { k-carragenano }\end{array}$ & RENDIMIENTO (\%) \\
\hline $\mathrm{N} .{ }^{\circ} 1(+)$ & $5,000 \mathrm{~g}$. & 1,8138 & 36,2 \\
\hline $\mathrm{N} .{ }^{\circ} 2\left({ }^{\Uparrow}\right)$ & $5,000 \mathrm{~g}$. & 1,7690 & 35,4 \\
\hline $\mathrm{N} .{ }^{\circ} 3(\otimes)$ & 4,6240 & 0,9445 & 20,4 \\
\hline $\mathrm{N} .{ }^{\circ} 4(4$ to $\mathrm{G})$ & 5,000 & 1,1475 & 23,0 \\
\hline
\end{tabular}

Tabla N. ${ }^{\circ}$ : Rendimiento de $\lambda$-carragenano

\begin{tabular}{|c|c|c|c|}
\hline MUESTRA & PESO MUESTRA & $\begin{array}{c}\text { PESO } \\
\lambda \text {-carragenano }\end{array}$ & RENDIMIENTO (\%) \\
\hline $\mathrm{N} .{ }^{\circ} 1(+)$ & $5,000 \mathrm{~g}$. & 0.8075 & 16,15 \\
\hline $\mathrm{N} .{ }^{\circ} 2\left({ }^{\curvearrowright}\right)$ & $5,000 \mathrm{~g}$. & 0.5233 & 10,5 \\
\hline $\mathrm{N} .{ }^{\circ} 3(\otimes)$ & 4,6240 & 2.0938 & 45,3 \\
\hline $\mathrm{N} .{ }^{\circ} 4(4$ to $\mathrm{G})$ & 5,000 & 0.7633 & 15,2 \\
\hline
\end{tabular}

\section{CARACTERIZACIÓN DE $\mathrm{k} Y$ A CARRAGENANOS}

La etapa de caracterización de $\mathrm{k}$ y $\lambda$ carragenanos se está efectuando actualmente por lo que no podemos adelantar resultados hasta que la caracterización finalice y sean comprobados. Los métodos de análisis que se están empleando corresponden a los siguientes:

\section{MÉTODO DE ANÁLISIS FÍSICO-QUÍMICOS}

- Determinación de la viscosidad de los carragenanos por el método Stormer o Brookfield; nos sirven para medir viscosidad y la consistencia de los geles.

- Determinación del punto de gelificación.

- Determinación del pH.

\section{MÉTODO DE ANÁLISIS QUÍMICOS}

- La caracterización de 3,6 anhidrogalactosa se determinará mediante el método espectrofotométrico de Yaphe y Arsenault (1965), con las modificaciones hechas por Craigie y Leigh (1978), empleando fructosa como estándar y 1,087 como factor de correc- 
ción. Lo que nos determinará la presencia de galactanos.

- El contenido de grupos sulfato se determinará por el método turbidimétrico, empleando cloruro de bario con sulfato de potasio como estándar.

- El grado de sulfatación (GS) se calculará mediante la razón $\mathrm{N} .^{\circ}$ de moles de sulfato $/ \mathrm{N} .^{\circ}$ moles de disacáridos.

Tabla N. ${ }^{\circ} 1$

Formulación de flan con k-carragenano obtenido

\begin{tabular}{|c|c|c|c|}
\hline & + & $\hat{0}$ & 4to. GRUPO \\
\hline Azúcar refinada & $29,16 \mathrm{~g}$ & 29,16 & 29,16 \\
\hline K-carragenano & 0,715 & 0,715 & 0,715 \\
\hline Sal refinada & 0,100 & 0,100 & 0,10 \\
\hline Etil vainillina & 0,018 & 0,018 & 0,018 \\
\hline Amarillo $\mathrm{N}^{\circ} 5$ & 0,0008 & 0,0005 & 0,0036 \\
\hline Rojo $\mathrm{N}^{\circ} 2$ & 0,0005 & 0,0004 & 0,0006 \\
\hline
\end{tabular}

Tabla N. ${ }^{\circ} 2$

Parámetros de control de flan

\begin{tabular}{|c|c|c|c|}
\hline & $\hat{\prime}$ & + & 4TO. GRUPO \\
\hline Tiempo de gelificación & $31^{\prime}$ & $26^{\prime}$ & $32^{\prime}$ \\
\hline Densidad & 1,0461 & 1.0458 & 1.0417 \\
\hline Color & MUY BUENO & BUENO & REGULAR \\
\hline Sabor & BUENO & MUY BUENO & MUY BUENO \\
\hline
\end{tabular}

Tabla N.$^{\circ} 3$

Formulación de pudín de chocolate

\begin{tabular}{|c|c|c|c|}
\hline & $\widehat{\lambda}$ & + & 4TO. GRUPO \\
\hline Azúcar refinada & $80,0 \mathrm{~g}$ & $80,0 \mathrm{~g}$ & $80,0 \mathrm{~g}$ \\
\hline K-carragenano & 1,0 & 1,0 & 1,0 \\
\hline Maizena & 5,0 & 5,0 & 5,0 \\
\hline Cocoa & 10,0 & 10,0 & 10,0 \\
\hline Etil vainillina & 0,2 & 0,2 & 0,2 \\
\hline Leche & $0,600 \mathrm{~L}$ & $0,600 \mathrm{~L}$ & $0,600 \mathrm{~L}$ \\
\hline
\end{tabular}

Tabla N..$^{\circ} 4$

Parámetros de control de pudín de chocolate

\begin{tabular}{|c|c|c|c|}
\hline & $\hat{0}$ & + & 4TO. GRUPO \\
\hline Tiempo de gelificación & $6^{\prime}$ & $5^{\prime}$ & $8^{\prime}$ \\
\hline Color & BUENO & BUENO & REGULAR \\
\hline Sabor & BUENO & MUY BUENO & BUENO \\
\hline
\end{tabular}

Tabla N. ${ }^{\circ} 5$

Formulación de leche chocolatada

\begin{tabular}{|c|c|}
\hline & Tetra, $\mathbb{\otimes}$ \\
\hline Azúcar refinada & $80,0 \mathrm{~g}$ \\
\hline Lambda carragenano & 0,5 \\
\hline Cocoa & 10,0 \\
\hline Ácido ascórbico & 0,030 \\
\hline Etil vainillina & 0,20 \\
\hline Sorbato de potasio & 0,24 \\
\hline Leche & $0,600 \mathrm{~L}$ \\
\hline
\end{tabular}

Tabla N. ${ }^{\circ} 6$

Parámetros de control de leche chocolatada

\begin{tabular}{|c|c|}
\hline ATRIBUTOS DE CALIDAD & Tetra, $\otimes$ \\
\hline Color & BUENO \\
\hline Sabor & MUY BUENO \\
\hline Consistencia & BUENA \\
\hline Textura & BUENA \\
\hline Dulzor & MUY ACENTUADO \\
\hline
\end{tabular}




\section{DISCUSIÓN}

\section{Fracción gelificante (k-carragenano) y Fracción no gelificante (lambda carragenano)}

La fracción gelificante es capturada en el filtro y tratada con cloruro de potasio formándose el gel consistente. El mecanismo de gelificación se presenta porque las moléculas de carragenano desarrollan estructuras helicoidales que reaccionan entre sí creando una red tridimensional.

De la Tabla $N .^{\circ} 3$ se observa que el mayor rendimiento en k-carragenano presenta la muestra que corresponde a fase gametofito femenino $(36,2 \%)$ seguida de la muestra de fase gametofito masculino $(35,4 \%)$.

La fracción que pasa a través del filtro, corresponde a la fracción no gelificante, siendo tratada con isopropanol coagulando completamente. De la Tabla $\mathrm{N} .{ }^{\circ} 4$ se desprende que el mayor rendimiento en lambda carragenano, corresponde a la fase esporofítica $(45,3 \%)$.

\section{Formulación de productos lácteos a partir de $\mathrm{k}$ y $\lambda$ carragenanos obtenidos}

La tecnología lechera presenta diversas formas de preparación de los productos lácteos cuidando su valor nutricional y sus propiedades sensoriales.

Los carragenanos interactúan favorablemente con las proteínas de la leche; en nuestro caso hemos diseñado la formulación de tres productos:

- Flan de leche formulado con k-carragenano obtenido.

- Pudín de chocolate con k-carragenano obtenido.

- Leche chocolatada con $\lambda$-carragenano obtenido.

El k-carragenano reemplazó a Lactogel FL 610 (flan) y Lactogel PS 451 (pudín) obteniéndose productos de excelente consistencia y con bajo contenido de calorías. Su preparación es simple no requiere de horneado ni tiempos de cocción prolongados.

La leche chocolatada presentó sabor, color, textura y consistencia muy buenos que confirma que la fase tetraspórica genera un carragenano que le imparte mayor viscosidad a las suspensiones.

Los flanes así obtenidos tienen menor contenido de calorías debido a que k-carragenano no aporta calorías que sí tiene el huevo. A medida que se enfría el flan va cuajando sin necesidad de refrigeración.

\section{Aplicaciones en la industria alimentaria}

- La capacidad gelificante (k-carragenano) y el comportamiento viscosante ( $\lambda$-carragenano) son características que los hacen útiles como estabilizantes o gelificantes de sistemas acuosos.

- Los carragenanos manifiestan un comportamiento reológico óptimo y cualidades organolépticas deseables.

- Las aplicaciones de k-carragenano como gelificante: postres lácteos, helados y otros.

- Milkshake, flanes y pudines: kappa le imparte buena consistencia

- Leche chocolatada: lambda mejora su textura.

- Crema de queso: kappa le da estabilidad y previene la sinéresis.

- Leche evaporada: Se emplea para evitar la separación de fases (k-carragenano)

- Alimentos para infantes: kappa estabiliza la grasa y las proteínas en formulaciones que emplean leche y/o soya.

- Yogurt: kappa actúa como estabilizante y mejora la consistencia.

\section{CONCLUSIONES}

- La identificación de las fases se realizó visualmente y químicamente mediante el método del resorcinol-acetal, que después de baño maría se observa la coloración y se concluye:

- Matiz rojo oscuro, corresponde a alga en fase de vida gametofita.

- Matiz color rosado, se trata de alga en fase de vida esporofita.

- Las unidades galactosa sulfatada en posición C6 han sido convertidas en unidades 3,6 anhidrogalactosas mediante tratamiento alcalino.

- De la Tabla N. ${ }^{0} 1$, se concluye que la fase tetraspórica presenta mayor proporción de carragenano semirrefinado y la mínima corresponde al 4to Grupo (mezcla de fases).

- La fracción gelificante se debe a las moléculas de k-carragenano que desarrollan estructuras helicoidales que reaccionan entre sí creando una red tridimensional. En la Tabla N. ${ }^{\circ} 3$, observamos el rendimiento de k-carragenano que alcanza al $36,2 \%$ para la fase gametofito femenino y $35,4 \%$ para la fase gametofito masculino.

- La fracción no gelificante (lambda carragenano) alcanza el mayor rendimiento $45,3 \%$ que corresponde a la fase tetraspórica como se observa en la Tabla N. ${ }^{\circ} 4$.

- La costa sur del Pacífico (Pisco) de aguas templadas posee condiciones climáticas muy especiales para el cultivo de estas algas (rodophitas), 
materia prima para la producción de hidrocoloides de múltiples aplicaciones en la Industria Alimentaria, Farmacéutica, Odontológica, Textil, Curtiembre.

\section{AGRADECIMIENTO}

Al Consejo Superior de Investigación de la UNMSM

\section{REFERENCIAS BIBLIOGRÁFICAS}

1. Bixler H., Kevin Johndro, Falshaw R. (2001). Kappa-2 carrageenan: Structure and performance of commercial extracts. Performance in two simulated dairy applications. Food Hidrocolloids. pp. 619-630.

2. Falshaw R., Bixler H.J., Johndro K. (2001). Structure and performance of commercial kappa - 2 carrageenan extracts. Food Hidrocolloids. pp. 441-452.

3. Falshaw R., Bixler H.J., Johndro K. (2003). Structure and performance of commercial K-2 carrageenan extracts. Part. III. Structure analysis and performance in two dairy applications of extracts from the New Zealand red Seaweed, Gigartina atropurpurea. Food Hidrocolloids. pp. 129-139.

4. López Acuña, L., M. (2002). Caracterización del carragenano de Chondracanthus pectinatus. Ciencias Marinas. pp. 311-318.

5. Bulboa C., Macchiavello J. (2006). Cultivo de Frondas cistocárpicas, tetraspóricas y vegetati- vas de Chondracanthus chamissoi en dos localidades del norte de Chile. Departamento de Biologia Marina. Facultad de Ciencias del Mar. Universidad Católica del Norte, Coquimbo, Chile.

6. Fuertes C. (1998). Polisacáridos sulfatados de algas marinas, elucidación estructural, actividad antiviral frente al virus del VIH. (Tesis). Facultad de Farmacia y Bioquímica. UNMSM.

7. Alama Vallejos E., Aojalla R. (2003). Estudio del efecto hipolipemiante de polisacáridos sulfatados de la fase tetraspórica del alga chondracanthus chamissoi en conejos (Tesis). Facultad de Farmacia y Bioquímica. UNMSM.

8. Suqueyama Mihagui H. L. (1978). Extracción de carragenina a partir del alga Gigartina chamissoi. (Tesis) Universidad Nacional Agraria La Molina.

9. Deza Durand K. (2002). Efecto del tamaño de corte sobre la tasa de crecimiento y cobertura de la microalga Chondracanthus chamissoi "yuyo" en Mendieta, Paracas. (tesis) Universidad Nacional Agraria La Molina.

10. Vandermeulen H. (1988). Crude extraction and testing of carrageenan. Huntsman Marine Laboratory. NB, Canada.

11. Ruperez P., Saura-Calixto F. (2001). Dietary and fibre physicochemical properties of edible spanish seaweeds. 